\title{
Reduced hardware architecture for energy-efficient IoT healthcare sensor nodes
}

\begin{abstract}
Healthcare solutions through the introduction of wearable healthcare devices are benefitting from Internet of Things technology. Though these small form-factor wearable devices promise great benefits, guaranteeing long device operating lifetime is yet the biggest challenge due to high-energy consumption. In this paper, a reduced hardware architecture system-on-chip targeting digital block design was proposed higher energy efficiency. The design has been verified by synthesizing into FPGA and implemented in silicon based on Silterra $180 \mathrm{~nm}$ process. Results show that the proposed design achieved reduction up to $24 \%$ of leakage power and $15 \%$ of dynamic power reduction over reference design. In addition, $24.3 \%$ of excessive area was reduced by using the proposed reduced hardware architecture technique.
\end{abstract}

Keyword: ASIC; Energy-efficient; FPGA; Internet of Things; Microcontroller 